\title{
Tratamento cirúrgico para automatismos sexuais em crises parciais complexas: relato de caso e revisão da literatura
}

Luiz Eduardo Ribeiro Wanderley Filho', Lucas Chaves Lelis', Caio Sander Andrade Portella Junior ${ }^{1}$, Yuri Andrade Souza², Vitor Andrade Souza ${ }^{3}$

Departamento de Neurocirurgia do Hospital São Rafael, Salvador, BA, Brasil.

\section{RESUMO}

Relatamos caso raro de crises parciais complexas com automatismos genitais secundárias a tumor temporal. Paciente foi submetida à lobectomia temporal direita com remissão completa dos sintomas. Após revisão da literatura, fica evidenciado que crises podem ser originadas tanto no lobo frontal quanto temporal.

\section{PALAVRAS-CHAVE}

Automatismo, lobo temporal, disfunção sexual fisiológica.

\begin{abstract}
Surgical treatment for sexual automatisms in complex partial seizures: case report and literature review

We report a rare case of sexual automatism in complex partial seizures secondary to temporal tumor. Patient underwent a right temporal lobectomy with complete remission of symptoms. After reviewing the literature, it is evident that the crisis can arise in both the frontal and temporal.
\end{abstract}

\section{KEYWORDS}

Automatism, temporal lobe, sexual dysfunction physiological. 


\section{Introdução}

Automatismos sexuais são manifestações raras durante ou após crises parciais complexas, em que há controvérsias em relação à localização anatômica do início da crise, com relatos da literatura de origem no lobo frontal e temporal. Trata-se de evento que tem recebido pouca importância na literatura, abrange atividade de preensão masturbatória, exibicionismo, hipermotricidade em movimentos pélvicos e de tronco ou outra atividade sexual para a qual o paciente é amnésico. ${ }^{1-3}$ Nosso objetivo é relatar caso de paciente do sexo feminino, tratada cirurgicamente devido a crises masturbatórias secundárias a ganglioglioma temporal direito, com revisão da literatura sobre o assunto.

\section{Relato de caso}

Paciente, feminina, 33 anos, com crises convulsivas parciais complexas há 11 anos, nega crises convulsivas febris na infância, traumatismo cranioencefálico, in- fecção do sistema nervoso central ou história familiar de epilepsia.

$\mathrm{Na}$ história clínica, familiares descrevem a crise como desconexões, seguidas de automatismo genitais com o membro superior direito, como ou sem generalização secundária. Paciente refere aura de Dejà vú e sensação epigástrica ascendente. Foi admitida em uso de carbamazepina (1.200 mg/dia), fenobarbital (200 mg/ dia) e clobazam ( $20 \mathrm{mg} / \mathrm{dia})$. Apresentava frequência de 3 a 5 crises diárias, com $20 \%$ apresentando generalização secundária.

$\mathrm{Na}$ investigação etiológica, ressonância magnética do crânio evidenciou formação expansiva intraxial cística na porção anterior do giro temporal inferior direito (Figura 1). Apresentava, ao eletroencefalograma interictal, ondas agudas em região temporal anterior (Figura 2A e B).

Paciente foi submetida à craniotomia pterional (Figura 3) com posterior lobectomia temporal anterior direita (Figura 4), sem intercorrências. Foram mantidas as mesmas medicações anticonvulsivantes por um ano, sem a presença de crises nesse período. Atualmente, em uso de fenobarbital $100 \mathrm{mg} / \mathrm{dia}$, apresenta vida social normal e retorno ao trabalho.

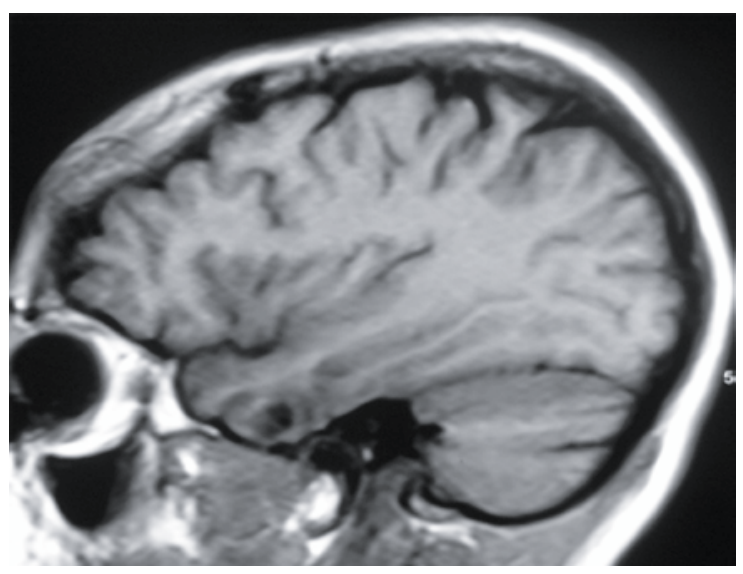

Figura 1 - Formação cística em giro temporal inferior direito.
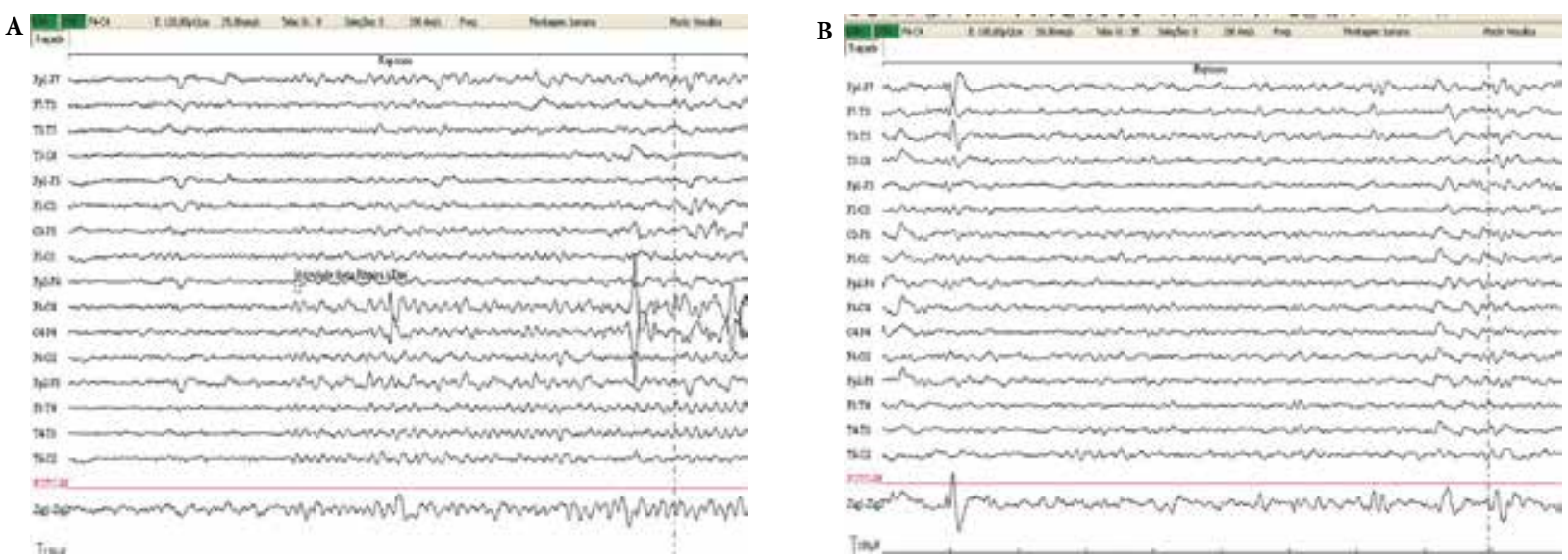

Figura 2 - (A, B) EEG com ondas agudas em região anterior do lobo temporal. 


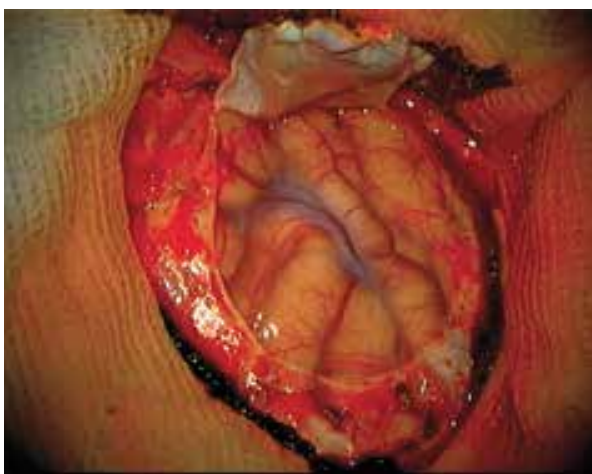

Figura 3 - Craniotomia pterional.

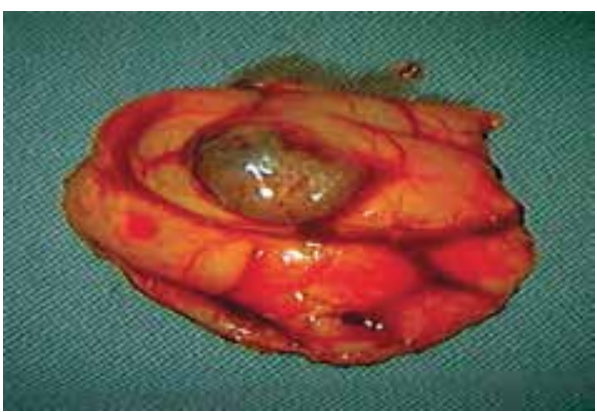

Figura 4 - Peça cirúrgica evidenciando a lesão.

\section{Discussão}

Manifestações sexuais são eventos incomuns ${ }^{4}$ durante ou após as crises parciais complexas que têm recebido pouca atenção na literatura. Podem apresentar-se como auras com conteúdo sexual, que têm sido relacionadas a atividade de apreensão com origem nos lobos temporais, sensações somatossensoriais nos órgãos genitais, relatada em pacientes com epilepsia do lobo parietal, acariciando e agarrando os órgãos genitais, assim como movimentos rítmicos de pelve e tronco. ${ }^{1-3}$

Os relatos sobre automatismos genitais são raros. Freemon e Nevis ${ }^{5}$ descrevem relato de paciente com crise parcial complexa que se iniciava com prurido e uma sensação de calor na região perineal e progressão para automatismos sexuais caracterizados por as pernas serem mantidas separadas, a paciente batendo no peito e as mãos manipulando o períneo. $O$ paciente foi amnésico para esses automatismos. EEG mostrou um F8 com foco de onda e a avaliação revelou uma artéria cerebral média direita hipoplásica. Convulsões foram controladas com primidona.

Em 1969, Hooshmand e Brawley ${ }^{6}$ relataram dois pacientes com manifestações exibicionistas de apreensão. Um paciente de 29 anos de idade, com uma longa história de expor seus órgãos genitais associada à confusão episódica, história de traumatismo craniano frontal, e picos na região F7 no EEG, onde as crises fo- ram controladas com medicação. O segundo paciente, de 32 anos, com automatismos genitais apresentava um astrocitoma estendendo-se para os lobos frontais e temporais. A lesão foi excisada com controle adequado das crises. Em 1971, Currier et al. ${ }^{7}$ fizeram o relato de três pacientes com comportamentos sexuais durante as crises conulsivas. Uma mulher de 52 anos, com crises em que ela levantava a saia, abduzia os membros inferiores e fazia movimentos rítmicos da pelve. EEG interictal mostrou picos de onda em região temporal esquerda, com convulsões controladas por medicação. Outra paciente, de 37 anos, apresentava crises tônicas em membro superior direito e dois episódios da atividade masturbatória relatados. EEG mostrou picos em região temporal anterior esquerda, realizou lesão estereotáxica na amígdala esquerda, com resolução das manifestações sexuais. Além disso, relata mulher de 50 anos, com aura orgásmica, na qual foi diagnosticado tumor hipotalâmico.

O significado de localização de automatismos sexuais é controverso. Spencer et al., ${ }^{8}$ em 1983, avaliou 61 pacientes com crises parciais complexas refratárias à medicação. Quatro desses pacientes apresentavam automatismos sexuais em algumas ou todas as suas crises convulsivas. A origem das crises epilépticas de início no lobo frontal foi documentada por EEG em três pacientes, os quais foram submetidos à lobectomia frontal com resolução de $75 \%$ a $100 \%$ das crises, em que os autores concluem que automatismos sexuais são originados nos lobos frontais.

Leutmezer et al., ${ }^{9}$ em 1999, avaliaram noventa pacientes com epilepsia focal refratária (74 de lobo temporal e 16 no lobo frontal ). A ocorrência dos automatismos sexuais foi avaliada durante o monitoramento de vídeo-EEG prolongado. Cinco pacientes apresentaram movimentos de preensão e acariciamento genital e todos os cinco tinham epilepsia do lobo temporal, evidenciada no vídeo-EEG. Os autores concluem que automatismos sexuais não podem ser exclusivos de crises no lobo frontal.

Concluímos que as manifestações sexuais representam fenômenos incomuns durante crises parciais complexas e não aparecem exclusivamente em crises do lobo frontal, como descrições anteriores. ${ }^{8}$ A definição detalhada do tipo de manifestação sexual pode ajudar no diagnóstico da localização do início da crise, pois essas parecem ser iniciadas tanto no lobo frontal como no temporal.

\section{Conflito de interesses}

Os autores declaram inexistência de conflito de interesses na realização deste trabalho. 


\section{Referências}

1. Remillard GM, Testa G, Anderman F, Feindel W, Gloor P, Martin JB. Sexual aura in seizures with partial complex symtomatology. In: Wada JA, Penry JK, editors. Advances in epileptology. The Tenth International Epilepsy Symposium. New York: Raven Press; 1980. p. 534-92.

2. Penfield W, Jasper H. Epilepsy and the functional anatomy of the human brain. London: J\&A Churchill; 1954.

3. York GK, Gabor AJ, Dreyfus PM. Paroxysmal genital pain: an unusual manifestation of epilepsy. Neurology. 1979;29(4):516-9.

4. Daly DD. Ictal clinical manifestations of complex partial seizures. Adv Neurol. 1975;11:57-83.

5. Freemon FR, Nevis AH. Temporal lobe sexual seizures. Neurology. 1969;19(1):87-90.
6. Hooshmand H, Brawley BW. Temporal lobe seizures and exhibitionism. Neurology. 1969;19(11):1119-24.

7. Currier RD, Little SC, Suess JF, Andy OJ. Sexual seizures. Arch Neurol. 1971;25(3):260-4.

8. Spencer SS, Spencer DD, Williamson PD, Mattson RH. Sexual automatisms in complex partial seizures. Neurology. 1983;33(5):527-33.

9. Leutmezer F, Serles W, Bacher J, Gröppel G, Pataraia E, Aull $\mathrm{S}$, et al. Genital automatisms in complex partial seizures. Neurology. 1999;52(6):1188-91.

Endereço para correspondência Luiz Eduardo Ribeiro Wanderley Filho

Av. Bosque imperial, bloco 11, ap. 203

41250-480 - Salvador, BA, Brasil

Email: luizeduardowand@yahoo.com.br 